facilities, policies and practices are designed along the gender binary and do not serve the healthcare needs of transgender women living with HIV. Positive aspects of care include support and the provision of appropriate services from community led and implemented interventions. Recommendations are made in relation to reactions to an HIV positive diagnosis, to support disclosure, address structural impediments in and outside of the health facilities. Recommendations are also made to support retention in care by addressing issues specifically to the health facility.

\section{S07.2 USING SURVEILLANCE DATA TO DETECT HIV CLUSTERS AND OUTBREAKS AND RESPOND TO ADDRESS GAPS IN PREVENTION}

A Oster*. Centers For Disease Control and Prevention, Atlanta, USA

10.1136/sextrans-2021-sti.35

Cluster detection and response is a long-standing public health approach to reducing illness and death from infectious diseases, but, until recently, has not been a routine part of HIV surveillance and prevention. The expansion of HIV cluster detection and response in the United States, including its role as one of four pillars of the U.S. federal Ending the HIV Epidemic initiative, provides an opportunity to identify rapid transmission so that proven, effective strategies can be implemented in affected populations or networks. HIV cluster detection uses multiple methods, including analysis of diagnosis data and molecular data submitted to HIV surveillance programs, to identify signals indicative or suggestive of rapid transmission. These surveillance-based methods, along with identification of clusters by partner services staff, health care providers, or community members, have varying strengths and are complementary. The presence of an HIV cluster or outbreak is a signal of increased HIV transmission within a geographic area, subpopulation, or social network, and indicates gaps in prevention or care. Responding to clusters and outbreaks involves understanding networks in which rapid transmission is occurring; linking people in the network to essential services; and identifying and addressing gaps in programs and services such as testing, HIV and other medical care, pre-exposure prophylaxis, and syringe services programs. We reviewed information disseminated through manuscripts, conference presentations, or public communications about more than 25 HIV cluster and outbreak responses in North America to identify approaches for implementing these core response strategies, and we provide key examples. HIV cluster detection and response provides a framework to guide tailored implementation of proven HIV prevention strategies where HIV transmission is occurring most rapidly and embodies the collaborative, data-guided approach that is critical to ending the HIV epidemic.

\section{SO7.4 TOOLS TO ENHANCE THE GLOBAL STI SURVEILLANCE}

T Wi* World Health Organization, Geneva, Switzerland

10.1136/sextrans-2021-sti.36

Without information, you don't have a problem. Without a problem, you can't advocate for resources. Without resources to tackle the problem, it grows bigger. However, without information, who would know?

There is a vicious cycle of limited STI surveillance and limited funding and responses, especially in resource-limited settings. Strengthened strategic information for STIs is needed to guide programming and service delivery, to rally political commitment and build a strong national investment case. National strategic information systems that incorporate STI case reporting, prevalence assessments, assessment of the etiologies of sexually transmitted infection syndromes, and monitoring for antimicrobial resistance to gonorrhoea are required.

Countries with STI surveillance systems rely on STI case reporting to estimate national incidence. Routine screening, currently most feasible for syphilis, address prevalence monitoring of priority populations.

Routine reporting and prevalence monitoring have limitations. STI reporting underestimates the burden of STIs as the majority are asymptomatic, limited access to care and challenges to collect verifiable data including limited diagnostics. Without diagnostics, it is difficult to count STI cases reliably. Periodic prevalence surveys using standard methods, increase confidence in trends by validating routine data with population denominators.

Specific studies including prevalence studies nested within DHS, biobehavioural surveys among key populations, a metaanalysis of STI data from other sources can enhance surveillance.

Tools are available to support STI surveillance. Modelling tools including Spectrum STI and the congenital syphilis estimation tool are available for national-level analysis of incidence and prevalence trends. Prevalence survey tools for pregnant women and enhanced gonorrhoea antimicrobial resistance surveillance programme protocol are available. A review of reporting tools is underway.

Striking the right balance of STI surveillance activities requires resources, building reporting systems and strengthening capacity at the national level to conduct and improve routine surveillance, while simultaneously investing in prevalence surveys and special studies.

\section{S08.1 DIAGNOSTIC INFECTIOUS DISEASES SELF-TESTING AND SELF-SAMPLING OUTSIDE CLINICS: A GLOBAL SYSTEMATIC REVIEW}

${ }^{1}$ E Kpokiri*, ${ }^{2} \mathrm{~W}$ Tang, ${ }^{3} \mathrm{G}$ Marley, ${ }^{1} \mathrm{~N}$ Fongwen, ${ }^{1} \mathrm{D}$ Wu, ${ }^{1} \mathrm{~S}$ Berendes, ${ }^{4} \mathrm{~B}$ Ambil, ${ }^{5} \mathrm{~S}$ Loveday, ${ }^{5} \mathrm{R}$ Sampath, ${ }^{6} \mathrm{~J}$ Walker, ${ }^{7} \mathrm{~J}$ Matovu, ${ }^{5} \mathrm{C}$ Boehme, ${ }^{8,9} \mathrm{~N}$ Pant Pai, ${ }^{1,10}$ J Tucker. ${ }^{1}$ London School of Hygiene and Tropical Medicine, London, UK; ${ }^{2}$ Southern Medical University, Guangzhou, China; ${ }^{3}$ Nanjing Medical University, Jiangsu, China; ${ }^{4}$ North Carolina State University, Raleigh, North Carolina, USA; ${ }^{5}$ Foundation for Innovative New Diagnostics, Switzerland; ${ }^{6}$ University of North Carolina, Chapel Hill, North Carolina, USA; ' Makerere University, Kampala, Uganda; ${ }^{8}$ CORE, Research Institute of McGill University, Montreal, Quebec, Canada; ${ }^{9}$ McGill University, Montreal, Quebec, Canada; ${ }^{10}$ University of North Carolina, Chapel Hill, North Carolina, USA

10.1136/sextrans-2021-sti.37

Most people around the world do not have access to facilitybased diagnostic testing and the gap in availability of diagnostic tests is a major public health challenge. Testing outside conventional clinical settings are transforming infectious disease diagnostic testing especially in low- and middle-income countries (LMICs). 
We conducted a systematic review and meta-analysis, searching six databases and including original research manuscripts comparing testing outside clinics with conventional testing. Main outcomes were test uptake and linkage to care, delivery models and adverse outcomes.

We identified 76 studies which were included. Data from 18 studies were pooled in meta-analyses. Studies focused on HIV (48), chlamydia (8), and multiple diseases (20). HIV selftesting increased test uptake compared with facility-based testing (nine studies, pooled OR $2.59,95 \% \mathrm{CI}=1 \cdot 06-6 \cdot 29$, moderate quality). STI self-sampling increased test uptake compared with facility-based testing (seven studies, pooled OR $1.7495 \% \mathrm{CI}=0.97-3.12$, moderate quality). Innovative and other digital test delivery services improved test uptake compared with facility-based testing services. We defined digital as emails, websites, instant messaging, or related internet approaches.

Our work shows testing outside of clinics increased test uptake without significant adverse outcomes. These testing approaches provide an opportunity to expand access and empower patients through innovative delivery models. From the policy viewpoint, this work demonstrates that the use of digital technology in testing outside clinics is a useful strategy for improving infectious diseases screening and linkage to care, and policies that aim to promote the use of these strategies are needed. These models may be especially useful for diseases associated with stigmatization such as HIV and other STIs in LMICs. However, inherent challenges include ensuring linkage to care continuum, quality assured testing and getting the right tests to the right people. Further implementation research and scale-up of effective decentralized models in LMIC settings is needed.

\section{S08.2 SELF-TESTING AND E-STI: THE DUTCH EXPERIENCE WITH HOME-BASED AND INTERNET-BASED SERVICES AND TRANSFORMATION INTO EFFECTIVE IMPLEMENTATION}

E Op de Coul*. Rivm, Bilthoven, The Netherlands

10.1136/sextrans-2021-sti.38

The main STI-healthcare providers in the Netherlands are GPs and sexual health clinics (SHCs). SHCs offer free-of-charge STI-care for key-populations. At the same time, Internet-based STI-test and home-sampling offers increase, offering new opportunities for a blended, more efficient, low threshold service. Currently, SHCs and national partners are reshaping sexual healthcare for young people and other key-populations, using a stepped-care model (SCM). The SCM is based on the principal that not all individuals need the same level of STIcare: some may be helped by online self-assessment tools (sense.info, Advies.chat) and a home sampling-kit and others need support by a professional.

NoMoreC and Limburg4zero are regional e-STI services for MSM, developed by SHCs, and co-created with MSM communities, healthcare professionals and other stakeholders, based on a scientific/theoretical framework. NoMoreC targets high-risk MSM for HCV-prevention and offers anonymous, low cost, home-sampled $\mathrm{HCV}$-testing. Limburg4zero provides home-sampling for HIV/STI testing as an extension of regular STI-care including peer-to-peer sharing, and e-counselling on e.g. chemsex and PrEP.
Opportunities

- Community involvement is essential to reach the right target groups

- Personalized e-STI advice and test-options provide a viable alternative for those preferring autonomic testing, and can saves costs

- The HCV-test service appeared especially suitable for MSM using PrEP who arrange their own STI-care

- e-STI should deliver prevention messages for those who tested negative

Challenges

- Quality assurance of test procedures (e.g. quality of the test, which test is offered) is a topic that should be high on the agenda

- Implementation is only effective when based on a clear theoretical/scientific framework

- Sustainability of regional e-STI initiatives is challenging; most are financed by research grants and broader/sustainable expansion may need governmental funding

- Tracking linkage-to-care is challenging in interventions with anonymous participation

- Return rates of home-sampling kits may be improved through digital innovations

\section{S08.3a HOW TO REALIZE IMPLEMENTATION IN HIV SELF- TESTING BY DISTRIBUTION VIA THE SOCIAL NETWORK IN CHINA: A JOINED COLLABORATION BETWEEN STAKEHOLDERS}

${ }^{1}$ W Tang ${ }^{*},{ }^{2} Y$ Zhou. 'University of North Carolina Project-China, Guangzhou, China; ${ }^{2}$ Zhuhai Center for Diseases Control and Prevention, Zhuhai, China

\subsection{6/sextrans-2021-sti.39}

HIV self-testing (HIVST) had been widely used in China since 2012, but social network strategies were rarely used to improve HIVST coverage. This presentation reports a case of community-based social network distribution of HIVST among Chinese men who have sex with men (MSM).

In 2016, UNC Project-China hosted an HIV testing intervention development designathon and invited eight teams from two provinces to contribute. The Zhuhai team designed an HIVST promotion proposal, which was a joint working plan between a community-based organization and the local CDC. They codeveloped a social media platform for self-test kits application and results reporting, which successfully provided 600 kits to MSM in 2016. However, due to the lack of evidence-based intervention, the program failed to increase the testing coverage and lack of innovation. After that, the researchers in the UNC Project-China team brought in secondary distribution idea, which allows the index MSM to apply for multiple kits and distribute them to people in their social network. After the piloting, 373 index MSM successfully motivated 287 alters for testing, among whole $40 \%$ were new testers, and the HIV testing yield among alters was significantly higher than that among index MSM. In the following RCT, a monetary incentive and peer referral further improved the mean number of kits that were distributed by index MSM $(\mathrm{MD}=1 \cdot 21,95 \% \mathrm{CI}: 0.77-1 \cdot 65)$. The strategy strongly increased testing coverage and was further implemented at the provincial level.

Opportunities

- Social-media platform provided an opportunity to reach a diverse index MSM 